disponer de la información adecuada para decidir si se debe invertir o no, por ejemplo, en sistemas y programas de detección precoz de esas enfermedades. Esto es particularmente importante en el caso del cáncer de próstata, no solamente por ser el segundo tipo de cáncer más frecuente en hombres, sino por los beneficios que pueden ofrecer su detección precoz y su oportuno tratamiento. No obstante, la efectividad de la detección temprana en la reducción de la mortalidad asociada con el cáncer de próstata es aún tema de debate.

Existen diversas estimaciones de los costos asociados con la detección, el diagnóstico y la clasificación del cáncer de próstata. Sin embargo, aún se desconocen los costos en recursos -el tiempo de trabajo del médico, los análisis de laboratorio, el tiempo que el paciente debe ausentarse del trabajo, entre otros - a tomar en cuenta para estas estimaciones.

En este trabajo se analizaron los costos de los recursos empleados en la detección, el diagnóstico y la clasificación por etapas del cáncer de próstata en los Estados Unidos de América y en qué medida esos costos difieren de los publicados en otros países industrializados.

Para ello se buscaron los artículos e informes sobre el cáncer de próstata publicados entre enero de 1980 y diciembre de 2003 e indizados en las bases de datos electrónicas MEDLINE, EMBASE y CINAHL. Se seleccionaron los artículos publicados en inglés, disponibles en texto completo, que contuvieran los datos sobre los costos de los recursos o los datos necesarios para estimar el costo de la detección, el diagnóstico y la clasificación de las etapas de cáncer de próstata. Se seleccionaron solamente los estudios realizados en países con economía de mercado consolidada. Para agrupar y analizar los datos obtenidos se utilizaron procedimientos estadísticos descriptivos, valores medios ponderados y el método de simulación Monte Carlo.

De los 262 estudios analizados, 28 cumplían con los criterios de selección (15 eran de los Estados Unidos y 13 de otros países industrializados). En los estudios realizados en los Estados Unidos se observó que el costo inicial combinado de los recursos para la detección mediante el antígeno prostático específico fue de US\$37,23 y para la detección mediante el examen rectal digital, de US\$31,77. En los estudios realizados en otros países industrializados, el costo inicial combinado de los recursos para la detección mediante el antígeno prostático específico fue de US\$ 30,92 y de US\$33,54 para la detección mediante el examen rectal digital. Se observó una gran variación en los costos de los recursos vinculados a los procedimientos de diagnóstico y de clasificación por etapas, tanto dentro de los Estados Unidos como en los otros países industrializados analizados.
Este estudio puede ser de gran utilidad para las autoridades sanitarias de otros países de la Región de las Américas, ya que por primera vez se ofrece un valor de base que puede emplearse como referencia al trazar políticas sanitarias relacionadas con el tamizaje - masivo o selectivo- de cáncer de próstata, siempre que se adapte a las condiciones locales. (Ekwueme DU, Stroud LA, Chen Y. Cost analysis of screening for, diagnosing, and staging prostate cancer based on a systematic review of published studies. Prev Chronic Dis. 2007;4(4). Hallado en http://www.cdc.gov/pcd/issues/2007/ oct/06_0051.htm. Acceso el 24 de enero de 2008.)

\section{Influencia de dos métodos de cálculo de las inequidades en salud: estudio en 22 países}

El efecto negativo de las inequidades socioeconómicas sobre la salud continúa siendo un tema prioritario en las agendas de las autoridades sanitarias de la mayoría de los países. Para los gobiernos y la comunidad internacional es importante y deseable poder medir y comparar la magnitud de las inequidades en salud - ya sea entre diferentes países o entre diferentes zonas o grupos de un mismo país-, así como evaluar la magnitud de los cambios ocurridos en esas inequidades en un período de tiempo dado.

Aunque existen muchas formas de evaluar el nivel de las inequidades en salud, las mediciones más empleadas son la razón de las tasas y la diferencia de las tasas. Estas mediciones se basan en la comparación (ya sea mediante la razón o la diferencia) de las tasas de mortalidad en relación con la posición socioeconómica. Para ello se comparan los valores de mortalidad correspondientes al segmento de mejor situación socioeconómica (por lo general el $20 \%$ superior) con la mortalidad correspondiente al segmento de peor situación socioeconómica (por lo general el 20\% inferior). A pesar de que estas mediciones no toman en cuenta toda la información disponible, son las más frecuentemente utilizadas por su sencillez y fácil uso.

En este trabajo se empleó información pública para analizar los niveles y las tendencias de la inequidad en salud a partir de los datos de mortalidad en menores de 5 años de 22 países clasificados como de ingresos bajos y mediano-bajos que aplicaron dos encuestas de población y salud entre 1991 y 2001. Se analizaron once países de África, seis de Asia y cinco de América Latina (Colombia, Guatemala, Haití, Nicaragua y Perú), que en su conjunto representan $27 \%$ de la población mundial.

Se observó que en ocasiones, la posición relativa de los países según su nivel de inequidad en un momento determinado varió en dependencia de la 
medición empleada, ya fuera la razón o la diferencia de las tasas (coeficiente de correlación de rangos de Spearman $=0,49$ ). De manera similar, la posición relativa de los países según la magnitud y la dirección de los cambios en los niveles de inequidad en el período de tiempo analizado varió también en dependencia del tipo de medición empleada. De gran importancia desde el punto de vista de la elaboración de políticas resultó el hecho de que la dirección de la tendencia observada mostró un cambio de signo (de aumento de la inequidad a la reducción) en cinco de los países (India, Namibia, Nepal, Perú y Vietnam) cuando se emplearon las dos mediciones estudiadas.

Esta es la primera comparación sistemática del uso de diferentes mediciones de la inequidad en salud, con herramientas estandarizadas, en un considerable número de países. El empleo indistinto de la razón de las tasas o de la diferencia de las tasas para medir la tendencia de los cambios en el nivel de inequidad generó diferencias sustanciales en el orden relativo que ocuparon los países analizados, tanto por la magnitud como por la dirección de los cambios. Estos resultados no se vieron afectados por factores de confusión - como el diseño del estudio, los métodos de obtención de los datos y los indicadores socioeconómicos, entre otros-y tienen importantes implicaciones para la elaboración de políticas, la atención sanitaria y la investigación.

A pesar de que no es posible hacer una recomendación categórica sobre las ventajas de medir la inequidad con métodos absolutos o relativos para comparar el efecto de las inequidades socioeconómicas en la salud, al analizar las inequidades y discutir las tasas se debe tomar en cuenta el método de medición utilizado, ya sea la razón de las tasas o la diferencia de las tasas, y el tipo de escala empleada, ya sea absoluta o relativa. (Moser K, Frost C, Leon DA. Comparing health inequalities across time and place - rate ratios and rate differences lead to different conclusions: analysis of cross-sectional data from 22 countries 1991-2001. Int J Epidemiol. 2007; 36:1285-91.)

\section{Estudio del Millón de Mujeres establece relación entre varios tipos de cáncer y el índice de masa corporal}

La prevalencia de la obesidad ha aumentado en el mundo. A pesar de que está ampliamente aceptado que el índice de masa corporal (IMC) está asociado directamente con el adenocarcinoma de esófago, el cáncer colorrectal, de endometrio y de riñón en la población general, y con el cáncer de mama en mujeres posmenopáusica, no se tienen datos exactos sobre la magnitud de este efecto y el papel que desempeña el IMC en el desarrollo de otros tipos de cáncer.

En este trabajo se investigó la relación entre la incidencia de y la mortalidad por 17 tipos/ubicaciones de cáncer y el IMC en las participantes en el denominado Estudio del Millón de Mujeres. Como parte de este estudio prospectivo de cohorte se realizó el seguimiento de 1,3 millones de mujeres de 50-64 años de edad del Reino Unido entre 1996 y 2001. El tiempo promedio de seguimiento fue de 5,4 años para la incidencia de cáncer y de 7,0 años para la mortalidad.

En el período de seguimiento se diagnosticaron 45037 casos de cáncer y ocurrieron 17203 muertes por esta causa. Se encontró una asociación significativa entre el aumento del IMC y el incremento en la incidencia de cáncer en 10 de las 17 ubicaciones específicas o tipos de cáncer estudiados: cáncer de endometrio (tendencia del riesgo relativo por 10 unidades $\left[\mathrm{tRR}_{10}\right]=2,89$; intervalo de confianza de 95\% [IC95\%]: 2,62 a 3,18), adenocarcinoma de esófago $\left(\mathrm{tRR}_{10}=2,38\right.$; IC95\%: 1,59 a 3,56), cáncer de riñón $\left(\mathrm{tRR}_{10}=1,53\right.$; IC95\%: 1,27 a 1,84), leucemia $\left(\mathrm{tRR}_{10}=1,50\right.$; IC95\%: 1,23 a 1,83), mielomas múltiples $\left(\mathrm{tRR}_{10}=1,31\right.$; IC95\%: 1,04 a 1,65$)$, cáncer de páncreas $\left(\mathrm{tRR}_{10}=1,24\right.$; IC95\%: 1,03 a $1,48)$, linfoma no Hodgkin $\left(\mathrm{tRR}_{10}=1,17\right.$; IC95\%: 1,03 a 1,34), cáncer de ovario $\left(\mathrm{tRR}_{10}=1,14 ; \mathrm{IC} 95 \%\right.$ : 1,03 a 1,27), todos los tipos de cáncer $\left(\operatorname{tRR}_{10}=1,12\right.$; IC95\%: 1,09 a 1,14), cáncer de mama en mujeres posmenopáusicas $\left(\mathrm{tRR}_{10}=1,40\right.$; $\mathrm{IC} 95 \%: 1,31$ a 1,49$)$ y cáncer colorrectal en mujeres premenopáusicas $\left(\mathrm{tRR}_{10}=1,61\right.$; IC95\%: 1,05 a 2,48). La asociación entre el IMC y la mortalidad por cáncer siguió un patrón similar al descrito para la incidencia.

El riesgo de cáncer de mama en mujeres premenopáusicas disminuyó a medida que aumentaba el IMC y aumentó con el IMC en las posmenopáusicas que no habían recibido tratamiento de sustitución hormonal. Estos datos demuestran que el estado de la mujer con relación a la menopausia constituye un factor clave en la relación entre el IMC y el riesgo de cáncer en mujeres, no solamente para los tipos de cáncer relacionados con las hormonas, como el cáncer de mama y de endometrio, sino también en otros tipos frecuentes de cáncer sin relación conocida con las hormonas. El efecto del IMC sobre el riesgo de melanomas malignos y cáncer colorrectal, de mama y de endometrio difiere significativamente según el estatus menopáusico de la mujer.

A pesar de la dificultad para lograr los estimados correspondientes a las mujeres que no han llegado a la menopausia con los datos disponibles, se concluyó que la proporción de casos de cáncer atribuibles al sobrepeso en mujeres premenopáusicas del Reino Unido puede ser menor que en mujeres posmenopáusicas, debido a la relación inversa 